\title{
Pronouns in competition: Predicting acquisition delays cross-linguistically ${ }^{1}$
}

\author{
Petra Hendriks, Irene Siekman, Erik-Jan Smits \& Jennifer Spenader \\ University of Groningen, The Netherlands
}

It is well known that English children between the age of 4 and 6 display a socalled Delay of Principle B Effect (DPBE) in that they allow pronouns to refer to a local c-commanding antecedent. Their guessing pattern with pronouns contrasts with their adult-like interpretation of reflexives. The DPBE has been explained as resulting from a lack of pragmatic knowledge or insufficient cognitive resources. However, such extra-grammatical accounts cannot explain why the DPBE only shows up in particular languages and in particular syntactic environments. Moreover, such accounts fail to explain why the DPBE only emerges in comprehension and not in production. This paper hypothesizes that the presence or absence of the DPBE can be explained from the properties of the grammar. Fischer's (2004) optimality-theoretic analysis of binding, explaining cross-linguistic variation, and Hendriks and Spenader's (2005/6) optimality-theoretic account of the acquisition of pronouns and reflexives are combined into a single model. This model yields testable predictions with respect to the presence or absence of the DPBE in particular languages, in particular syntactic environments, and in comprehension and/or production.

\section{Introduction}

According to standard Binding Theory (cf. Chomsky, 1981), the distribution and interpretation of reflexives is regulated by Principle A, whereas the distribution and interpretation of pronouns is regulated by Principle B.
(1) Principle A:
Reflexives must be bound in their binding domain.
Principle B:
Pronouns must be free in their binding domain.

1 Petra Hendriks gratefully acknowledges the Netherlands Organisation for Scientific Research, NWO (grant no. 277-70-005). 
Assuming that the binding domain is the entire clause, using himself in (2) in production to refer to the subject Bert satisfies Principle A, whereas using him to refer to the subject is ruled out by Principle B, as is illustrated by (3). In comprehension, Principle A explains why the reflexive himself in (2) must be interpreted as co-referential with the subject Bert and cannot refer to some other individual. Similarly, Principle B accounts for the fact that the pronoun him in (3) cannot be co-referential with the subject and must refer to some other individual.

(2) Bert $t_{\mathrm{i}}$ washed himself $\mathrm{f}_{\mathrm{i} / *_{\mathrm{j}}}$

(3) Berti washed him $*_{i j}$

The formulation of Principle A and Principle B in (1) suggests that reflexives and pronouns are always in complementary distribution. However, several exceptions have been observed to the general pattern of complementary distribution:

(4) Bert $\mathrm{i}_{\mathrm{i}}$ saw a snake near himself $\mathrm{i} / \mathrm{him}_{\mathrm{i} / \mathrm{j}}$

In (4), both a reflexive and a pronoun are allowed in a locational PP, but such a breakdown of complementarity has also been observed for other constructions, for example picture NPs.

For Reinhart and Reuland (1993), these exceptions motivated replacing the binding principles by syntactic and semantic conditions on reflexivity. Fischer (2004), in contrast, maintains one of the binding principles but argues that this principle must be violable rather than strict, in order to account for cases such as (4). In the next section, Fischer's optimality-theoretic approach to binding will be discussed. This approach allows Fischer to account for the broad range of cross-linguistic variation that is encountered in the field of binding. However, Fischer's optimality-theoretic analysis differs in several respects from Hendriks and Spenader's (2005/6) optimality-theoretic analysis of binding, discussed in section 3, which aims at accounting for the acquisition delay observed with pronouns. In section 4 , the two analyses are integrated into one model. Section 5 presents the predictions of the resulting model with respect to crosslinguistic variation in language acquisition. In particular, predictions will be formulated with respect to the occurrence of the so-called Delay of Principle B effect in English, Dutch, German and Italian. 


\section{Optimal binding}

Fischer's (2004) analysis of binding is couched in the framework of Optimality Theory (OT) syntax (cf., e.g., Prince and Smolensky, 2004). In OT syntax, starting from an input meaning, the Generator generates a candidate set consisting of all possible forms for this input meaning. These candidate forms are then evaluated on the basis of a universal set of violable constraints. A crucial property of OT constraints is that they are ordered in a hierarchy of strength: If two constraints are in conflict, it is more important to satisfy the stronger constraint than it is to satisfy the weaker constraint. The candidate that satisfies the total set of constraints best is the optimal candidate. All other candidates are ungrammatical.

\subsection{Constraint sub-hierarchies}

Constraints in OT can be part of universal constraint sub-hierarchies (cf. Aissen, 1999). According to Fischer (2004), the distribution of pronouns and reflexives across languages can be explained through the interaction between two potentially conflicting constraint sub-hierarchies. The first sub-hierarchy favours binding in local domains and punishes binding in bigger domains. The second sub-hierarchy punishes the occurrence of certain forms in the output.

The first sub-hierarchy favours binding within the smallest domain possible. The smallest domain is the Theta Domain, which is the smallest phrase containing the head that theta-marks the anaphor plus its arguments. An example is the PP near himself/him in (4). The somewhat bigger Subject Domain is the smallest phrase containing the anaphor and a subject. Examples are the sentences (2), (3) and (4). The Root Domain is the entire sentence containing the anaphor. The Root Domain allows Fischer to distinguish between an embedded clause (the Subject Domain) and the entire sentence containing the embedded clause (the Root Domain). See Fischer (2004) for definitions of these domains as well as for the additional domains Case Domain, Finite Domain, and Indicative Domain. Note that the smaller domain is always included in the bigger domain. For example, in (4) the Theta Domain near himself/him is included in the Subject Domain and the Root Domain, which both comprise the entire sentence.

Principle A is modified by Fischer (2004) to be sensitive to these binding domains of different size, resulting in a family of Principle A constraints with the ranking as given in (5). ${ }^{2}$ The constraints within this sub-hierarchy are or-

2 Actually, Fischer (2004) introduces this family of constraints as Principle B constraints, but then goes on to revise them according to the definition given in the text, referring to the resulting constraints as Reflexivity constraints. Because the constraints, as they are formulated, specify the conditions under which a form occurs bound rather than free, they 
dered in strength from left to right. In effect, binding within the smallest Theta Domain is preferred to binding within the bigger Subject Domain, which is again preferred to binding within the biggest Root Domain. In general, principles referring to smaller domains are universally higher-ranked than those that refer to bigger domains.

$$
\begin{aligned}
& \text { Universal sub-hierarchy } 1 \text { (Fischer, partial): } \\
& \text { Principle } \mathrm{A}_{\text {Theta Domain }}>>\text { Principle } \mathrm{A}_{\text {Subject Domain }}>>\text { Principle } \mathrm{A}_{\text {Root Domain }}
\end{aligned}
$$

A second modification of the binding principles by Fischer involves their sensitivity to different degrees of anaphoricity. For example, Principle $A_{\text {Subject Domain }}$ is defined as follows (p. 492): "If $\alpha$ is bound in its subject domain, $\alpha$ must be maximally anaphoric". ${ }^{3}$ Principle $A_{\text {Subject Domain }}$ and Principle $A_{\text {Root Domain }}$ are defined similarly. In these definitions, $\alpha$ is a reflexive or a pronoun, and reflexives are more anaphoric than pronouns.

The second sub-hierarchy necessary to account for cross-linguistic variation in binding prefers pronouns to reflexives. Furthermore, within the class of reflexives this sub-hierarchy prefers SE anaphors (i.e., morphologically simplex reflexives, such as Dutch zich) to SELF anaphors (i.e., morphologically complex reflexives, such as English himself). This effect is obtained by assuming that *SELF (read: No SELF anaphors) is stronger than *SE, which is again stronger than *Pronoun.

(6) Universal sub-hierarchy 2 (Fischer, partial):

*SELF $>>$ *SE $>>$ *Pronoun

According to Fischer, "the hierarchy can be interpreted as an indication of the decrease in anaphoricity" (2004: 491). That is, if the speaker maximally satisfies this sub-hierarchy, he is as explicit as possible and selects forms that are minimally anaphoric. Thus this sub-hierarchy conflicts with the first sub-hierarchy,

show more resemblance to standard Principle A than to standard Principle B. For this reason, we will refer to them as Principle A constraints.

3 This formulation of Principle Asubject Domain and Fischer's other Principle A constraints is somewhat problematic from an OT perspective because the evaluation of a candidate output with respect to this constraint is dependent on the evaluation of other candidate outputs with respect to the same constraint. To determine whether $\alpha$ is the maximally anaphoric element satisfying this constraint, it must be established that there is no candidate output higher in anaphoricity that also satisfies this constraint. For each candidate higher in anaphoricity, one constraint violation is counted. A fundamental assumption of OT, however, is that candidates are compared on the basis of the evaluation function rather than on the basis of the constraints themselves (McCarthy, 2002: 40). 
which prefers the output form to be maximally anaphoric. The two universal constraint sub-hierarchies in (5) and (6) are part of the constraint hierarchy that forms the grammar of a language.

\subsection{Constraint re-ranking and tied constraints}

A fundamental assumption within OT is the assumption that languages share the same set of constraints but differ in the ranking of these constraints. Differences between languages can be explained through a different ranking of the same set of constraints ('typology by re-ranking'). This suggests that the different possibilities for interleaving the two constraint sub-hierarchies introduced in the previous section, giving rise to different grammars, may explain cross-linguistic differences with respect to binding.

Constraints can be stronger or weaker than other constraints. In addition, two constraints can be tied. Fischer includes the option of tied constraints to account for the optionality illustrated by sentence (4), where both a reflexive and a pronoun are possible as prepositional objects. If two constraints $\mathrm{X}$ and $\mathrm{Y}$ are tied (notation: $\mathrm{X}^{\circ} \mathrm{Y}$ ), a violation of $\mathrm{X}$ is as serious as a violation of $\mathrm{Y}$. Crucially, a constraint hierarchy with a tie between two constraints is in fact short-hand for two separate hierarchies, one of them containing the dominance relation $\mathrm{X}>\mathrm{Y}$ and the other containing the dominance relation $\mathrm{Y}>>\mathrm{X}$. A tie between two constraints often yields more than one optimal candidate.

\subsection{Binding in English}

As a first example of the interaction between the constraint sub-hierarchies introduced in section 2.1, let us consider the case of English. According to Fischer's (2004) analysis, the constraint ranking in English is as in (7):

(7) English ranking (Fischer):

Principle $\mathrm{A}_{\mathrm{TD}} \gg>* \mathrm{SELF}{ }^{\circ}$ Principle $\mathrm{A}_{\mathrm{SD}} \gg>* \mathrm{SE}>>*$ Pronoun

For reasons of simplicity, we omit Principle $A_{\text {Root Domain }}$ (which is stronger than

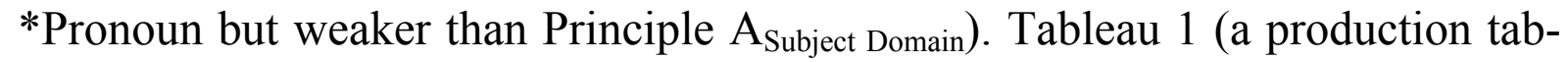
leau) illustrates the selection of the optimal form in example (2)/(3). 
Tableau 1: Selection of the optimal form in "[SD [TD Bert $t_{i}$ washed $\left.\left.-_{i}\right]\right]$ ", where the direct object is co-referential with the subject, according to the analysis of Fischer (2004).

\begin{tabular}{|l|c|cc|c|c|}
\hline \multicolumn{1}{|c|}{ Production } & Principle $\mathrm{A}_{\mathrm{TD}}$ & *SELF & Principle $\mathrm{A}_{\mathrm{SD}}$ & *SE & *Pronoun \\
\hline himself & & $*$ & & & \\
\hline $\operatorname{him}$ & $* !$ & & $*$ & & $*$ \\
\hline
\end{tabular}

In sentence (2), the Theta Domain is the same as the Subject Domain, namely the entire sentence. All constraints, ranked as in (7), apply to select the optimal candidate. Candidate outputs are the reflexive himself and the pronoun him. English does not have SE anaphors (but see section 4.3 for a discussion of SE versus SELF anaphors in Dutch and German). Because English does not have $\mathrm{SE}$ anaphors, the constraint $* \mathrm{SE}$ is never violated. Selection of the pronoun him results in a violation of Principle $\mathrm{A}_{\mathrm{TD}}$ as well as Principle $\mathrm{A}_{\mathrm{SD}}$ (indicated by an asterisk in the corresponding cell). This is because a pronoun is not the maximally anaphoric element that is bound within these domains. The more anaphoric reflexive is also bound within these domains. Selecting the reflexive himself violates the constraint $* \mathrm{SELF}$, whereas selecting a pronoun violates the constraint *Pronoun. However, these two constraint violations do not matter here because violation of the strongest constraint Principle $\mathrm{A}_{\mathrm{TD}}$ by the pronoun already yields the reflexive as the optimal candidate. Such a fatal violation is indicated by an exclamation mark. Optimal candidates are indicated by the pointing hand $(\varpi)$.

The proposed constraint ranking is also able to account for the optionality in (4), as illustrated by Tableau 2.

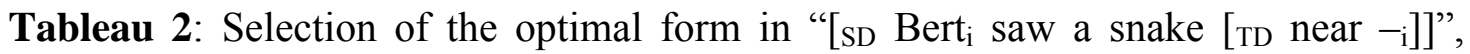
where the prepositional object is co-referential with the subject, according to the analysis of Fischer (2004).

\begin{tabular}{|l|c|cc|c|c|}
\hline \multicolumn{1}{|c|}{ Production } & Principle $\mathrm{A}_{\mathrm{TD}}$ & *SELF & Principle $\mathrm{A}_{\mathrm{SD}}$ & $* \mathrm{SE}$ & *Pronoun \\
\hline himself & & $*$ & & & \\
\hline $\mathrm{him}$ & & & $*$ & & $*$ \\
\hline
\end{tabular}

In sentence (4), the Theta Domain is the PP, whereas the Subject Domain is the entire sentence. Both himself and him satisfy Principle $A_{\text {TD }}$ because $\alpha$ is not bound in its Theta Domain. Because *SELF and Principle $A_{S D}$ are tied constraints, both the ranking *SELF $>>$ Principle $A_{S D}$ and the ranking Principle $A_{S D}$ $>{ }^{*}$ SELF should be considered. If *SELF is ranked higher than Principle $\mathrm{A}_{\mathrm{SD}}$, him is the optimal candidate because himself violates the stronger constraint *SELF. If Principle $\mathrm{A}_{\mathrm{SD}}$ is ranked higher, himself is the optimal candidate be- 
cause him violates Principle $\mathrm{A}_{\mathrm{SD}}$ (himself is a more anaphoric form bound in the Subject Domain). As a result of *SELF and Principle $A_{\mathrm{SD}}$ being tied, both himself and him come out as optimal and hence are possible forms for expressing the input meaning in the sentence under discussion.

\subsection{Explaining differences between English, German, Dutch, Italian, and Icelandic}

Different rankings of the constraint sub-hierarchies proposed in section 2.1 give rise to different distributions of pronouns and reflexives.

German has two different types of reflexives: the SE anaphor sich and the SELF anaphor sich selbst. Fischer (2004) observes that in German a pronoun is not allowed in sentences like (4). In this case, German uses a SE anaphor. The use of a SELF anaphor seems only marginally acceptable. According to Fischer, this pattern can be explained under the assumption that the constraint *SELF is not tied with Principle $A_{S D}$, as in English, but with the higher ranked constraint Principle $A_{T D}$.

German ranking (Fischer):

Principle $\mathrm{A}_{\mathrm{TD}}{ }^{\circ} * \mathrm{SELF}>>$ Principle $\mathrm{A}_{\mathrm{SD}}>>* \mathrm{SE}>>*$ Pronoun

The German pattern more or less falls out of this constraint ranking, as Tableau 3 shows, although the proposed constraint ranking does not explain why a SELF anaphor seems slightly more acceptable than a pronoun.

Tableau 3: Selection of the optimal form in the German sentence "[ $\mathrm{sD}_{\text {Bert }}$ bemerkte eine Schlange [TD neben -i]]" (Bert saw a snake near -), where the prepositional object is co-referential with the subject, according to the analysis of Fischer (2004).

\begin{tabular}{|l|cc|c|c|c|}
\hline Production & Principle $\mathrm{A}_{\mathrm{TD}}$ & *SELF & Principle $\mathrm{A}_{\mathrm{SD}}$ & $* \mathrm{SE}$ & *Pronoun \\
\hline SELF & & $* !$ & & & \\
\hline $\mathrm{SE}$ & & $*$ & $*$ & \\
\hline pronoun & & $* * !$ & & $*$ \\
\hline
\end{tabular}

Like German, Dutch also distinguishes a SE anaphor, zich, and a SELF anaphor, zichzelf. However, in Dutch a pronoun as well as a SE anaphor is allowed in sentences like (4), whereas a SELF anaphor is unacceptable, according to Fischer. The Dutch ranking explaining this pattern is characterized by a tie between Principle $A_{S D}$ and $*$ SE. 


$$
\begin{aligned}
& \text { Dutch ranking (Fischer): } \\
& \text { Principle } \mathrm{A}_{\mathrm{TD}}>>* \mathrm{SELF}>>\text { Principle } \mathrm{A}_{\mathrm{SD}}{ }^{\circ} * \mathrm{SE}>>* \text { Pronoun }
\end{aligned}
$$

Italian also distinguishes between the $\mathrm{SE}$ anaphor (which can be realized as a clitic si or a full form sé) and the SELF anaphor se stesso. According to Fischer (fn. 16), whether the elements in Italian occur as clitics or full forms is regulated by different constraints and is not subject to the Binding Theory. In Italian, like German, a SE anaphor is used in sentences like (4). A SELF anaphor is unacceptable in this context, as in Dutch, and a pronoun is almost acceptable. The Italian constraint ranking explaining this pattern is characterized by two ties: a tie between Principle $A_{\mathrm{TD}}$ and ${ }^{*} \mathrm{SELF}$ and a tie between Principle $\mathrm{A}_{\mathrm{SD}}$ and ${ }^{*} \mathrm{SE}$.

(10) Italian ranking (Fischer):

Principle $\mathrm{A}_{\mathrm{TD}}{ }^{\circ} * \mathrm{SELF}>>$ Principle $\mathrm{A}_{\mathrm{SD}}{ }^{\circ} * \mathrm{SE}>>*$ Pronoun

This ranking predicts that in Italian both a pronoun and a SE anaphor are possible in sentences like (4).

All languages discussed above allow a reflexive in a locational PP to be bound by the subject. A language showing even longer distance binding is Icelandic:

(11) Jón ${ }_{\mathrm{i}}$ skipaði Pétrij $\mathrm{PRO}_{\mathrm{j}}$ að raka sig $\mathrm{j}_{\mathrm{i}}$ ??sjálfan sig $\mathrm{i}_{\mathrm{i}} / \mathrm{hann}_{\mathrm{i}}$ á hverjum degi.

John ordered Peter to shave-Inf SE/himself/him on every day

"John ${ }_{\mathrm{i}}$ ordered Peter to shave him $_{\mathrm{i}}$ every day"

As this example shows, in Icelandic a SE anaphor can be bound outside its Subject Domain. This can be explained by a Principle A constraint referring to the Root Domain. The constraint ranking explaining Long Distance Anaphora is the following: ${ }^{4}$

(12) Icelandic ranking (Fischer, partial):

$* \mathrm{SELF}>>$ Principle $\mathrm{A}_{\mathrm{RD}}{ }^{\circ} * \mathrm{SE}>>*$ Pronoun

Thus Fischer is able to explain cross-linguistic differences in binding between languages through the different inventory (e.g., English does not have SE anaphors) in combination with a different ranking of the same set of constraints. In the next section, we will look at the acquisition of the binding principles. An

4 The reader is referred to Fischer (2004) for a more detailed analysis of Icelandic, as well as an account of differences between e.g. Icelandic, Faroese, and Russian. 
important question is whether the constraints introduced in this section are able to explain the acquisition pattern observed with binding.

\section{Optimal acquisition of the binding principles}

If children learn the grammar of their language, one of the things they must acquire is the correct ranking of the universal set of constraints. On the basis of the constraints discussed in the previous section, it is expected that pronouns are not more difficult to learn than reflexives because pronouns and reflexives are subject to the same set of Principle A constraints. Also, there is no reason to believe that *Pronoun should be more difficult to learn than *SE or *SELF. Furthermore, without any additional assumptions, it is expected that learning to comprehend pronouns is as easy as learning to produce pronouns. The same set of constraints can be used for production and comprehension, in the same way the standard binding principles regulate the distribution and interpretation of pronouns and reflexives. If the child knows the constraints and has established the correct ranking between these constraints, it is expected that (s)he displays the adult pattern of production and comprehension.

However, with respect to the binding principles a remarkable pattern can be observed in language acquisition. Whereas children correctly interpret reflexives from a young age on, they display difficulties correctly interpreting pronouns until the age of 6 or 7 (see, e.g., Chien and Wexler, 1990). This delay between the correct comprehension of reflexives and the correct comprehension of pronouns is known as the Delay of Principle B Effect (DPBE). Children displaying the DPBE allow for a co-referential interpretation for pronouns in simple transitive sentences such as (3) about half of the time, which seems to be the result of chance performance. The DPBE has been explained as resulting from a lack of pragmatic knowledge (Thornton and Wexler, 1999) or insufficient cognitive resources (Reinhart, in press). Another remarkable pattern in the acquisition of pronouns, which has only recently received attention, concerns the difference between production and comprehension. In contrast with their performance in comprehension, children's production of both pronouns and reflexives is adultlike from a young age on (de Villiers, Cahillane, and Altreuter, 2006).

These two asymmetries (the one between reflexives and pronouns, and the other between production and comprehension) are explained by Hendriks and Spenader $(2005 / 6)$ by assuming that children initially use the grammar in one direction only. Children optimize from meaning to form in production (as in OT syntax), and from meaning to form in comprehension (as in OT semantics). To arrive at the adult pattern of binding, however, Hendriks and Spenader argue that language users also have to learn to take into account the opposite perspective (as in bidirectional OT). As a hearer, they must consider the alternative 
forms a speaker could have used. And as a speaker, they must consider the alternative meanings a hearer may assign to the produced form. By taking into account the opposite perspective in communication, one of the meanings can be blocked for potentially ambiguous forms such as pronouns. This yields the adult pattern of production and comprehension. This mechanism of bidirectional optimization will be discussed in section 3.2 below. But first we will look at children's pattern of production and comprehension, which can be modelled as unidirectional optimization from meaning to form or from form to meaning.

\subsection{Unidirectional optimization}

To account for children's pattern as well as the adult pattern, Hendriks and Spenader (2005/6) employ the following constraints under the ranking given.

\section{(13) English ranking (Hendriks and Spenader): \\ Principle A $>$ * Pronoun $>>*$ Reflexive}

Because they consider only simple transitive sentences such as (2) and (3), a single Principle A constraint ("A reflexive must be bound locally") suffices. Two differences between Fischer's (2004) constraint ranking of English and the constraint ranking in (13) are relevant. First, Hendriks and Spenader's constraint Principle A does not require comparing different anaphoric expressions, which seems to be an improvement on Fischer's constraint family Principle A (see fn. 3 ). Second, the ranking of *Pronoun and *Reflexive is the exact opposite of the ranking in Fischer's account of binding, but is compatible with Burzio's (1998) constraint sub-hierarchy Referential Economy.

The three constraints in (13) account for the production of reflexives as in Tableau 4, and for the production of pronouns as in Tableau 5. In production, the input is a meaning, and the grammar selects the optimal form for expressing that meaning.

Tableau 4: Selection of the optimal form in "[SD [TD Bert $t_{\mathrm{i}}$ washed $\left.-_{\mathrm{i}}\right]$ ]", where the direct object is co-referential with the subject, according to the analysis of Hendriks and Spenader $(2005 / 6)$.

\begin{tabular}{|l|l|c|c|}
\hline Production & Principle A & *Pronoun & $*$ Reflexive \\
\hline reflexive & & & $*$ \\
\hline pronoun & & $* !$ & \\
\hline
\end{tabular}


Tableau 5: Selection of the optimal form in "[SD [TD Bert $t_{\mathrm{i}}$ washed $\left.\left.-_{\mathrm{j}}\right]\right]$ ", where the direct object is disjoint to the subject, according to the analysis of Hendriks and Spenader (2005/6).

\begin{tabular}{|l|c|c|c|}
\hline Production & Principle A & $*$ Pronoun & $*$ Reflexive \\
\hline reflexive & $* !$ & & $*$ \\
\hline pronoun & & $*$ & \\
\hline
\end{tabular}

The resulting pattern is identical to the pattern resulting from Fischer's constraints. In comprehension, however, the constraints in (13) give rise to a different pattern. Tableau 6 shows the comprehension of reflexives, and Tableau 7 shows the comprehension of pronouns.

Tableau 6: Selection of the optimal meaning for the sentence "[SD [TD Bert washed himself]]", according to the analysis of Hendriks and Spenader (2005/6).

\begin{tabular}{|l|c|c|c|}
\hline Comprehension & Principle A & *Pronoun & $*$ Reflexive \\
\hline co-referential meaning & & & $*$ \\
\hline disjoint meaning & $* !$ & & $*$ \\
\hline
\end{tabular}

Tableau 7: Selection of the optimal meaning for the sentence "[ $\mathrm{SD}_{\text {[TD }}$ Bert washed him]]", according to the analysis of Hendriks and Spenader (2005/6).

\begin{tabular}{|l|l|c|l|}
\hline Comprehension & Principle A & $*$ Pronoun & *Reflexive \\
\hline$*$ co-referential meaning & & $*$ & \\
\hline$*$ disjoint meaning & & $*$ & \\
\hline
\end{tabular}

In comprehension, the form is given as the input. As a result, all candidate meanings for this form violate the constraints *Pronoun and *Reflexive to the same degree. Therefore, Principle A is crucial in deciding on the optimal meaning. Because a disjoint meaning (Bert $t_{i}$ washed himself $f_{j}$ ) violates Principle $A$, a co-referential meaning $\left(\right.$ Bert $_{i}$ washed himself $\left.f_{i}\right)$ is the optimal meaning for the reflexive. Crucially, a pronoun never violates Principle A, irrespective of the selected meaning. Principle A prevents a reflexive form from expressing a disjoint meaning ${ }^{5}$, but does not restrict the interpretation of pronouns. As a result, for a pronoun both a disjoint and a co-referential meaning are optimal. The two meanings will each be chosen half of the time, which corresponds to children's guessing pattern with pronouns. Thus the pattern resulting from unidirectional

5 The constraint Principle A can thus be seen as prohibiting the association between a reflexive form and a disjoint meaning, and could also be formulated as: Avoid reflexive forms with a disjoint meaning. 
optimization from meaning to form and from form to meaning is exactly the pattern of production and comprehension displayed by children.

\subsection{Bidirectional optimization}

According to Hendriks and Spenader (2005/6), adults not only optimize from meaning to form or from form to meaning, but also take into account the opposite perspective in communication, a mechanism known as bidirectional optimization. ${ }^{6}$ According to the constraint ranking in (13), reflexives are preferred to pronouns because *Pronoun is stronger than *Reflexive. The optimal formmeaning pair, satisfying the constraints of the English grammar best, therefore is the pair consisting of a reflexive form and a co-referential meaning (which is the optimal meaning for this form according to Tableau 6). This optimal pair blocks the reflexive as the form for expressing other, less harmonic, meanings, and also blocks the co-referential meaning for other, less harmonic, forms. So although pronouns are potentially ambiguous in comprehension, the co-referential meaning is blocked for the pronoun because this meaning is better expressed using a reflexive. Consequently, for adults, who optimize bidirectionally, pronouns must be disjoint to the subject. This mechanism of bidirectional optimization is illustrated by Tableau 8 . Bidirectionally optimal pairs are indicated by the symbol $\mathrm{e}$. Blocked pairs are indicated by the X. Note that the grammar (i.e., the constraints and their ranking) is the same as in previous tableaux. Only the mechanism of optimization (bidirectional rather than unidirectional) is different.

Tableau 8: Selection of the optimal form-meaning pair for the sentence "[SD [TD Bert washed -]]", according to the analysis of Hendriks and Spenader (2005/6).

\begin{tabular}{|l|c|c|c|}
\hline \multicolumn{1}{|c|}{ Bidirectional } & Principle A & $*$ Pronoun & $*$ Reflexive \\
\hline < $<$ reflexive, co-referential meaning $>$ & & & $*$ \\
\hline $\mathrm{X}<$ reflexive, disjoint meaning $>$ & $*$ & & $*$ \\
\hline $\mathrm{X}<$ pronoun, co-referential meaning $>$ & & $*$ & \\
\hline < $<$ pronoun, disjoint meaning $>$ & & $*$ & \\
\hline
\end{tabular}

6 Bidirectional optimization is defined over form-meaning pairs (Blutner, 2000). A formmeaning pair $<\mathrm{f}, \mathrm{m}>$ is bidirectionally optimal iff:

a. there is no other pair $\left\langle\mathrm{f}^{\prime}, \mathrm{m}\right\rangle$ such that $\left\langle\mathrm{f}^{\prime}, \mathrm{m}>\right.$ is more harmonic than $\langle\mathrm{f}, \mathrm{m}\rangle$.

b. there is no other pair $<$, m' $>$ such that $\langle$ f,m' $>$ is more harmonic than $<$, $m>$.

The more harmonic pair is the pair that satisfies the constraints of the grammar best. A bidirectionally optimal pair is a pair for which there is no pair with the same meaning but a better form (condition a), nor a pair with the same form but a better meaning (condition b), given the constraints of the grammar. A bidirectionally optimal pair blocks other pairs with either the same form or the same meaning. 
Acquisition delays arise as a result of a different output for unidirectional versus bidirectional optimization. Under unidirectional optimization, pronouns can be assigned both a disjoint and a co-referential meaning. Under bidirectional optimization, pronouns are only interpreted as disjoint with the subject. As a result, children are expected to interpret pronouns differently than adults and hence display a comprehension delay. Because unidirectional and bidirectional optimization yield the same results in production, no acquisition delay arises in production. Thus Hendriks and Spenader (2005/6) explain why children's correct production of pronouns precedes their comprehension.

Although the binding account of Hendriks and Spenader (2005/6) accounts for children's and adults' pattern of forms and meanings in simple transitive sentences in English, their account does not predict any cross-linguistic differences, nor does it explain the cases where both a pronoun and a reflexive are allowed. To account for the acquisition data as well as cross-linguistic differences, we seem to need a combination of Hendriks and Spenader's analysis and Fischer's analysis. In the next section, we will investigate the possibilities for integrating the two analyses into a single model of optimization.

\section{$4 \quad$ Integrating the two analyses}

\subsection{Principle A hierarchy}

Both Fischer (2004) and Hendriks and Spenader (2005/6) proceed from Principle A and derive the effects of Principle B from the interaction of Principle A and a markedness hierarchy. Accordingly, we will also assume a family of Principle A constraints, sensitive to binding domains of different size. Principle A constraints are formulated as follows: Principle $A_{\text {Subject Domain: "A reflexive must }}$

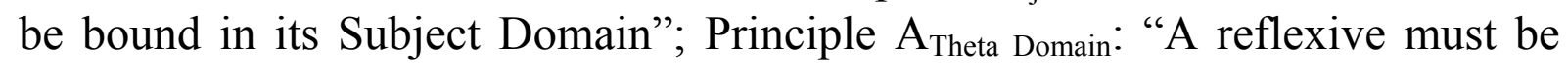
bound in its Theta Domain"; etc. This formulation has the advantage that the evaluation of a candidate with respect to these constraints is not dependent on the evaluation of other candidates (cf. fn. 3). Whether a reflexive violates or satisfies this constraint can be determined without looking at whether other candidate outputs violate or satisfy this constraint.

\subsection{Markedness hierarchy}

According to Fischer's (2004) analysis, pronouns are preferred to reflexives. On the other hand, Hendriks and Spenader's (2005/6) markedness hierarchy on referential forms assumes that reflexives are preferred to pronouns. Fischer motivates her choice by stating (p. 487) that her preference has to counterbalance the effects of Principle A. Hendriks and Spenader's choice for the opposite preference is motivated by the observation that reflexive meanings are somehow easier to learn than pronominal meanings. If pronouns were the preferred forms, 
reflexives are incorrectly predicted to be acquired last or even not acquired at all.

However, there is a way to combine these two opposing views on markedness. If reflexives are assumed to be preferred to pronouns (cf. Hendriks and Spenader, 2005/6), while at the same time bigger binding domains are preferred to smaller binding domains (which is the reverse of Fischer's hierarchy), the effects of the markedness hierarchy on referential forms counterbalance the effects of Principle A. So rather than assuming that binding within the smallest Theta Domain is preferred to binding within the bigger Subject Domain, we assume that binding within the bigger Subject Domain is preferred to binding within the smaller Theta Domain. This preference is reflected in the sub-hierarchy in (14):

$$
\begin{aligned}
& \text { Universal sub-hierarchy } 1 \text { (revised): } \\
& \text { Principle } A_{\text {Root Domain }}>>\text { Principle } A_{\text {Subject Domain }}>>\text { Principle } A_{\text {Theta Domain }}
\end{aligned}
$$

Simultaneously, we assume that reflexives are preferred to pronouns:

(15)

*Pronoun $>>$ *Reflexive

In section 4.4 below, we show that the interaction between these two subhierarchies yields the correct pattern for English. Moreover, the interaction between these two sub-hierarchies is also able to explain the observed pattern of language acquisition in English.

Thus far, we have only talked about reflexives in general, without distinguishing between SE anaphors and SELF anaphors. In the next section, we take a closer look at the distinction between SE anaphors and SELF anaphors.

\subsection{SE versus SELF}

Fischer (2004) splits up the constraint *Reflexive into the two more fine-grained constraints *SELF and *SE to explain the different distribution of SE anaphors and SELF anaphors in German and Dutch. Fischer notes that in Dutch a SE anaphor is excluded in sentences like (17), whereas in its German counterpart (18) both a SE anaphor and a SELF anaphor are possible.

(16) Bert $_{\mathrm{i}}$ hates himself $\mathrm{i} /{ }^{*}$ him $_{\mathrm{i}}$

(17) Bert $_{\mathrm{i}}$ haat zichzelf $\mathrm{i} /{ }^{*} \mathrm{zich}_{\mathrm{i}} / *^{*}$ hem $_{\mathrm{i}}$

(18) Bert $_{\mathrm{i}}$ hasst sich selbst $/ \mathrm{sich}_{\mathrm{i}} / *_{\mathrm{ihn}}$ 
According to Fischer (2004: fn. 15), the difference between (17) and (18) is an illustration of the general tendency that the German reflexives sich and sich selbst are more interchangeable than Dutch zich and zichzelf. She explains this from a different constraint ranking for Dutch and German, which results in partly differing binding domains for zich/zichzelf and sich/sich selbst. However, Geurts (2004), among others, shows that the choice between zich and zichzelf in Dutch may be influenced by semantic and pragmatic factors such as habitualness of the action and focus, rather than by syntactic factors such as binding domains. Moreover, the division of labour between Dutch zich and zichzelf may be explainable in the same terms as the division of labour between German sich and sich selbst. The German SELF anaphor has been argued to be the result of reanalysis of the string consisting of a SE anaphor and an intensifying particle (Eckardt, 2001), which explains the focus behaviour and the distribution of sich selbst. Geurts shows that the focus properties of Dutch zelf are like those of German selbst in all relevant respects, making a similar analysis of Dutch zich/zichzelf and German sich/sich selbst plausible.

In addition, Smits, Hendriks, and Spenader (2007) show in their study of the distribution of Dutch zich and zichzelf in the Clef corpus (a 70 million word large parsed corpus of Dutch) that the choice between zich and zichzelf is tendential rather than categorical. Many transitive verbs can occur with zich as well as zichzelf as the direct object; even inherently reflexive verbs sometimes occur with zichzelf. The choice of zich versus zichzelf was found to be strongly correlated with the frequency with which the verb is used with reflexive and nonreflexive events. The ratio with which a verb is used to describe events acted to others correlates with the degree to which the verb prefers zichzelf, and the ratio with which a verb is used to describe events acted to oneself correlates with the degree to which the verb prefers zich. Since the tendencies are related to the types of real world events the verb describes, the choice between zich and zichzelf can hardly be ascribed to binding domains.

For these reasons, we hypothesize that the constraint *Reflexive suffices for an adequate account of binding. The distinction between SE anaphors and SELF anaphors must be regulated by semantic or pragmatic constraints unrelated to Binding Theory. An additional advantage of avoiding such a distinction in our binding constraints is that we do not need to make Principle A sensitive to different degrees of anaphoricity (see fn. 3).

\subsection{A revised optimality-theoretic model of binding}

Taking into account the above considerations, we end up with the following constraint ranking for English: 
(19) English ranking (revised):

Principle $\mathrm{A}_{\text {Subject Domain }}>>*$ Pronoun ${ }^{\circ}$ Principle $\mathrm{A}_{\text {Theta Domain }}>>*$ Reflexive

According to the universal sub-hierarchies introduced in section 4.2, Principle $\mathrm{A}_{\text {Subject Domain }}$ is stronger than Principle $\mathrm{A}_{\text {Theta Domain, }}$ and *Pronoun is stronger than *Reflexive. In English, the constraints *Pronoun and Principle $A_{\text {Theta Domain }}$ are tied.

Now let us look at the unidirectional production and comprehension of reflexives and pronouns in simple transitive sentences, according to our revised optimality-theoretic model. A co-referential meaning is best expressed using a reflexive (Tableau 9). A disjoint meaning is best expressed using a pronoun (Tableau 10).

Tableau 9: Selection of the optimal form in "[SD [TD Bert ${ }_{i}$ washed $\left.\left.-_{i}\right]\right]$ ", where the direct object is co-referential with the subject, according to the revised model.

\begin{tabular}{|l|l|l|c|}
\hline Production & Pr. $\mathrm{A}_{\mathrm{SD}}$ & *Pronoun Pr. $\mathrm{A}_{\mathrm{TD}}$ & *Reflexive \\
\hline reflexive & & & $*$ \\
\hline pronoun & & $* !$ & \\
\hline
\end{tabular}

Tableau 10: Selection of the optimal form in "[SD [TD Bert $t_{i}$ washed $\left.\left.-{ }_{j}\right]\right]$ ", where the direct object is disjoint to the subject, according to the revised model.

\begin{tabular}{|l|c|cc|c|}
\hline Production & Pr. $\mathrm{A}_{\mathrm{SD}}$ & *Pronoun & Pr. $\mathrm{A}_{\mathrm{TD}}$ & *Reflexive \\
\hline reflexive & $* !$ & & $*$ & $*$ \\
\hline pronoun & & $*$ & & \\
\hline
\end{tabular}

This is also the adult pattern of production. In comprehension, on the other hand, the unidirectional model yields a different pattern than the bidirectional model. Under unidirectional optimization, the optimal meaning for a reflexive is a coreferential one (Tableau 11). For pronouns, under unidirectional optimization two optimal meanings emerge: a co-referential meaning and a disjoint meaning (Tableau 12).

Tableau 11: Selection of the optimal meaning for the sentence "[ $\mathrm{SD}$ [TD Bert washed himself]]", according to the revised model.

\begin{tabular}{|l|c|cc|c|}
\hline Comprehension & Pr. $\mathrm{A}_{\mathrm{SD}}$ & *Pronoun & Pr. $\mathrm{A}_{\mathrm{TD}}$ & $*$ Reflexive \\
\hline co-referential meaning & & & $*$ \\
\hline disjoint meaning & $* !$ & $*$ & $*$ \\
\hline
\end{tabular}


Tableau 12: Selection of the optimal meaning for the sentence "[ $\mathrm{SD}$ [TD Bert washed him]]", according to the revised model.

\begin{tabular}{|l|c|c|c|}
\hline Comprehension & Pr. $\mathrm{A}_{\mathrm{SD}}$ & *Pronoun Pr. $\mathrm{A}_{\mathrm{TD}}$ & *Reflexive \\
\hline \multirow{2}{|c|}{ co-referential meaning } & & $*$ & \\
\hline disjoint meaning & & $*$ & \\
\hline
\end{tabular}

Adults, however, will not assign a co-referential meaning to a pronoun in comprehension because they also take into account production. In production, a coreferential meaning is best expressed using a reflexive. Hence, the co-referential meaning is blocked for the pronoun. As a result, under bidirectional optimization a pronoun is interpreted as expressing a disjoint meaning only (Tableau 13).

Tableau 13: Selection of the optimal form-meaning pair for the sentence "[ $\mathrm{SD}$ [TD Bert washed -]]", according to the revised model.

\begin{tabular}{|c|c|c|c|c|c|}
\hline & Bidirectional & Pr. $A_{S D}$ & *Pronoun & Pr. $A_{T D}$ & *Reflexive \\
\hline 急 & $<$ reflexive, co-referential meaning $>$ & & & & $*$ \\
\hline $\mathrm{X}$ & $<$ reflexive, disjoint meaning $>$ & $*$ & & $*$ & $*$ \\
\hline $\mathrm{X}$ & $<$ pronoun, co-referential meaning $>$ & & $*$ & & \\
\hline 斗 & $<$ pronoun, disjoint meaning $>$ & & * & & \\
\hline
\end{tabular}

So bidirectional optimization yields a different interpretation for pronouns than unidirectional optimization does.

Unidirectional optimization nicely describes children's pattern of production and comprehension. In contrast, the adult pattern of production and comprehension is best described by the mechanism of bidirectional optimization. So let us proceed from the assumption that children optimize unidirectionally whereas adults optimize bidirectionally. On the basis of the revised constraints and their ranking we thus predict a DPBE in simple transitive sentences in English. Consequently, our revised model yields the same predictions with respect to the acquisition of reflexives and pronouns as Hendriks and Spenader's (2005/6) model. In the next section, we will see whether the revised model is also able to generate new predictions with respect to the acquisition of pronouns in other syntactic environments than simple transitive sentences and in other languages than English.

\section{$5 \quad$ Predicting acquisition delays}

In this section, we will look at the predictions of our revised model. Recall that a Delay of Principle B Effect arises if, under the same constraints and their ranking, bidirectional optimization and unidirectional optimization yield different 
outputs. Section 5.1 considers locational PPs in English. Section 5.2 focuses on embedded clauses in English and the other languages under discussion. In section 5.3, the predictions of our model are discussed for Dutch transitive sentences, and in section 5.4 for Dutch locational PPs. In sections 5.5 and 5.6 we present the predictions for German locational PPs and German transitive sentences, respectively. Section 5.7, finally, discusses transitive sentences in Italian. Because there is some disagreement with respect to the relevant acceptability judgments for Icelandic pronouns (see Fischer, 2004: fn. 21), we will omit Icelandic from the present discussion.

\subsection{No DPBE in English locational PPs}

Unidirectional optimization from meaning to form predicts that co-reference between the subject and the argument of a locational preposition can be expressed by a reflexive as well as a pronoun (Tableau 14).

Tableau 14: Selection of the optimal form in "[SD Bert ${ }_{i}$ saw a snake [TD near $\left.-{ }_{i}\right]$ ]", where the prepositional object is co-referential with the subject, according to the revised model.

\begin{tabular}{|l|c|cc|c|}
\hline \multicolumn{1}{|l|}{ Production } & Pr. $\mathrm{A}_{\mathrm{SD}}$ & *Pronoun & Pr. $\mathrm{A}_{\mathrm{TD}}$ & *Reflexive \\
\hline reflexive & & & $*$ & $*$ \\
\hline pronoun & \multicolumn{3}{|c|}{$*$} & \\
\hline
\end{tabular}

A disjoint meaning must be expressed using a pronoun (Tableau 15):

Tableau 15: Selection of the optimal form in "[SD Bert ${ }_{i}$ saw a snake [TD near $\left.-{ }_{j}\right]$ ]", where the prepositional object is disjoint to the subject, according to the revised model.

\begin{tabular}{|l|c|cc|c|}
\hline \multicolumn{1}{|c|}{ Production } & Pr. $\mathrm{A}_{\mathrm{SD}}$ & *Pronoun & Pr. $\mathrm{A}_{\mathrm{TD}}$ & *Reflexive \\
\hline reflexive & $* !$ & & $*$ & $*$ \\
\hline \multirow{2}{*}{ pronoun } & \multicolumn{3}{|c|}{$*$} \\
\hline
\end{tabular}

In comprehension, a pronoun is predicted to be interpreted as co-referential with the subject as well as disjoint to the subject (Tableau 16). As the reader can check for himself, a reflexive is predicted to be co-referential with the subject (no tableau is given here). 
Tableau 16: Selection of the optimal meaning for the sentence "[sD Bert saw a snake [TD near him]]", according to the revised model.

\begin{tabular}{|l|c|c|c|}
\hline Comprehension & Pr. $\mathrm{A}_{\mathrm{SD}}$ & *Pronoun Pr. $\mathrm{A}_{\mathrm{TD}}$ & *Reflexive \\
\hline co-referential meaning & & $*$ & \\
\hline disjoint meaning & & $*$ & \\
\hline
\end{tabular}

Bidirectional optimization yields three optimal form-meaning pairs (Tableau 17):

Tableau 17: Selection of the optimal form-meaning pair for the sentence "[SD Bert saw a snake [TD near - ]]”, according to the revised model.

\begin{tabular}{|c|c|c|c|c|c|}
\hline & Bidirectional & Pr. $A_{S D}$ & *Pronoun & Pr. $A_{T D}$ & *Reflexive \\
\hline 为 & $<$ reflexive, co-referential meaning $>$ & & & $*$ & $*$ \\
\hline $\mathrm{X}$ & $<$ reflexive, disjoint meaning $>$ & $*$ & & $*$ & $*$ \\
\hline 为 & $<$ pronoun, co-referential meaning $>$ & & $*$ & & \\
\hline 为 & $<$ pronoun, disjoint meaning $>$ & & $*$ & & \\
\hline
\end{tabular}

Because children's unidirectional interpretation of pronouns is identical to adults' bidirectional interpretation of pronouns (compare Tableau 16 and Tableau 17; in both cases, pronouns are ambiguous), no DPBE is predicted for pronouns in locational PPs in English. This prediction may be generalized to all other cases in English where the Theta Domain is smaller than the Subject Domain (e.g. in picture NPs). Because, both under unidirectional production and under bidirectional production, co-reference between the subject and the argument of a locational preposition can be optionally expressed by a reflexive or a pronoun, no production delay is predicted either.

\subsection{No DPBE in English embedded clauses}

Consider the following example, where a reflexive cannot be bound by the subject of the matrix clause, and a pronoun should be used instead to express coreference with the matrix subject. The same pattern can be observed in German, Dutch and Italian.

*Bert ${ }_{\mathrm{i}}$ knows that Mary washed himself $\mathrm{i}_{\mathrm{i}}$

Because the local subject Mary differs from the reflexive in gender, the reflexive cannot be bound by the local subject. Strictly speaking, we would require a higher-ranked constraint on agreement of gender features between the anaphor and the antecedent to block the local binding relation. However, for reasons of 
clarity, we omit this constraint from the tableaux below. A bidirectional tableau gives the correct results:

Tableau 18: Selection of the optimal form-meaning pair for the sentence " $[\mathrm{RD}$ Bert knows that [SD [TD Mary washed - ]]]", according to the revised model.

\begin{tabular}{|c|c|c|c|c|c|}
\hline & Bidirectional & Pr. $A_{S D}$ & *Pronoun & Pr. $A_{T D}$ & *Reflexive \\
\hline $\mathrm{X}$ & $<$ reflexive, co-referential meaning $>$ & $*$ & & $*$ & $*$ \\
\hline $\mathrm{X}$ & $<$ reflexive, disjoint meaning $>$ & * & & * & $*$ \\
\hline 急 & $<$ pronoun, co-referential meaning $>$ & & $*$ & & \\
\hline (3) & $<$ pronoun, disjoint meaning $>$ & & $*$ & & \\
\hline
\end{tabular}

As Tableau 18 shows, a pronoun is the preferred form in (20). This pronoun can be co-referential with the matrix subject, but can also be disjoint to the matrix subject, provided that pronoun and antecedent agree in gender features.

As the reader can check for himself, children's pattern of production of pronouns and reflexives is adult-like. However, an interesting pattern emerges from children's unidirectional comprehension of sentence (20). Although this sentence is ungrammatical, the sentence can nevertheless be assigned a meaning, as is the case for all ungrammatical input in Optimality Theory:

Tableau 19: Selection of the optimal meaning for the sentence "[ ${ }_{\mathrm{RD}}$ Bert knows that [SD [TD Mary washed himself]]]", according to the revised model.

\begin{tabular}{|l|c|cc|c|}
\hline Comprehension & Pr. $\mathrm{A}_{\mathrm{SD}}$ & *Pronoun & Pr. $\mathrm{A}_{\mathrm{TD}}$ & $*$ Reflexive \\
\hline co-referential meaning & $*$ & $*$ & $*$ \\
\hline disjoint meaning & $*$ & $*$ & $*$ \\
\hline
\end{tabular}

According to Tableau 19, children will be able to assign both a co-referential meaning and a disjoint meaning to the reflexive in (20).

Does this mean that children's comprehension of (20) differs from the adult comprehension of this sentence? Do we predict a DPBE in this case? As Tableau 18 shows, for adults reflexives are never the optimal form in (20). However, if adults would hear a reflexive in the syntactic environment in (20), the constraints of their grammar would not be able to distinguish between a coreferential meaning and a disjoint meaning. According to Tableau 18, a coreferential meaning for a reflexive (the first candidate) and a disjoint meaning for a reflexive (the second candidate) violate and satisfy the same constraints. This is in fact the same constraint profile as in the unidirectional comprehension Tableau 19. Hence, the grammar predicts no DPBE for reflexive objects in embedded clauses in English. Because Principle $A_{\text {Subject Domain }}$ is high-ranked in all 
languages under consideration, as we will see below, the same prediction holds for German, Dutch and Italian.

\subsection{A DPBE in Dutch transitive sentences}

If the division of labour between SE anaphors and SELF anaphors is not determined by the markedness sub-hierarchy of referential forms, as was argued in section 4.3, the same constraints that play a role in binding in English should also play a role in Dutch. Consequently, the pattern of acquisition of binding in Dutch is predicted to be the same as in English. That is, a DPBE is also predicted for transitive sentences in Dutch. For Dutch, this prediction has been experimentally confirmed (Koster, 1993; Philip and Coopmans, 1996). The comprehension delay in Dutch has been observed for pronouns versus the SELF anaphor zichzelf, as well as for pronouns versus the SE anaphor zich.

\subsection{No DPBE in Dutch locational PPs}

Assuming that the same constraint ranking is responsible for binding in English and Dutch, our model also predicts the same pattern of acquisition for locational PPs in these languages. That is, for Dutch, as for English (cf. section 5.1), no DPBE is predicted for pronominal objects in locational PPs. As yet, however, we are not aware of any study investigating the acquisition of pronominal reference in locational PPs.

\subsection{A DPBE in German locational PPs}

Fischer (2004: 494) observes that German differs from English and Dutch in that it does not allow pronouns in locational PPs to be co-referential with the subject:

(21) $B$ Bert $t_{\mathrm{i}}$ bemerkte eine Schlange neben sich $\mathrm{h}_{\mathrm{i}} /$ ??sich selbst $\mathrm{i}_{\mathrm{i}} * \mathrm{ihm}_{\mathrm{i}}$

Bert ${ }_{i}$ noticed a snake next.to $\mathrm{SE}_{i} / \mathrm{SELF}_{i} /$ him $_{i}$

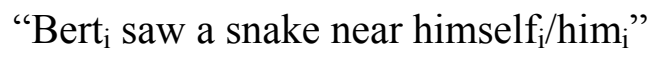

This can be modelled by assuming that in German the constraint *Pronoun must be stronger than the constraint Principle $\mathrm{A}_{\mathrm{TD}}$.

Tableau 20: Selection of the optimal form in the German sentence "[sD Bert bemerkte eine Schlange [TD neben -i]]" (Bert saw a snake near-), where the prepositional object is co-referential with the subject, according to the revised model.

\begin{tabular}{|l|c|c|c|c|}
\hline Production & Pr. $\mathrm{A}_{\mathrm{SD}}$ & *Pronoun & Pr. $\mathrm{A}_{\mathrm{TD}}$ & *Reflexive \\
\hline reflexive & & & $*$ & $*$ \\
\hline pronoun & & $*$ & & \\
\hline
\end{tabular}


Under this ranking, a reflexive (sich or sich selbst) is the optimal form in production (Tableau 20). In comprehension, a pronoun will be ambiguous between a co-referential and a disjoint meaning (Tableau 21). Tableaux 20 and 21 illustrate unidirectional optimization, which models the pattern of production and comprehension for children.

Tableau 21: Selection of the optimal meaning for the German sentence "[sD Bert bemerkte eine Schlange [TD neben ihn]]" (Bert saw a snake near him), according to the revised model.

\begin{tabular}{|l|l|c|l|l|}
\hline Comprehension & Pr. $\mathrm{A}_{\mathrm{SD}}$ & $*$ Pronoun & Pr. $\mathrm{A}_{\mathrm{TD}}$ & $*$ Reflexive \\
\hline co-referential meaning & & $*$ & & \\
\hline disjoint meaning & & $*$ & & \\
\hline
\end{tabular}

The pattern of production and comprehension for adults is modelled by bidirectional optimization. Because the co-referential meaning is blocked under bidirectional optimization (Tableau 22), a DPBE is predicted in locational PPs in German.

Tableau 22: Selection of the optimal form-meaning pair for the German sentence "[SD Bert bemerkte eine Schlange [TD neben -]]" (Bert saw a snake near-), according to the revised model.

\begin{tabular}{|c|c|c|c|c|c|}
\hline & Bidirectional & Pr. $A_{S D}$ & *Pronoun & Pr. $A_{T D}$ & *Reflexive \\
\hline 尚 & $<$ reflexive, co-referential meaning $>$ & & & $*$ & $*$ \\
\hline $\mathrm{X}$ & $<$ reflexive, disjoint meaning $>$ & $*$ & & $*$ & $*$ \\
\hline $\mathrm{X}$ & $<$ pronoun, co-referential meaning $>$ & & $*$ & & \\
\hline 尚 & $<$ pronoun, disjoint meaning $>$ & & $*$ & & \\
\hline
\end{tabular}

Note that this analysis, and the prediction of a comprehension delay, hinges on the unacceptability of the pronoun in the syntactic environment in (21). If pronouns turn out to be acceptable in this syntactic environment after all, the German adult pattern can be explained in the same way as the Dutch pattern, and no DPBE is predicted.

\subsection{A DPBE in German transitive sentences}

Adopting the constraint ranking in the previous section for German, object pronouns in transitive sentences are predicted to be ambiguous between a coreferential and a disjoint meaning: 
Tableau 23: Selection of the optimal meaning for the German sentence "[SD [TD Bert hasst ihn]]" (Bert hates him), according to the revised model.

\begin{tabular}{|l|c|c|c|c|}
\hline Comprehension & Pr. $\mathrm{A}_{\mathrm{SD}}$ & *Pronoun & Pr. $\mathrm{A}_{\mathrm{TD}}$ & *Reflexive \\
\hline co-referential meaning & & $*$ & & \\
\hline disjoint meaning & & $*$ & & \\
\hline
\end{tabular}

Because the co-referential meaning for the pronoun is blocked by bidirectional optimization (Tableau 24), and hence children's unidirectional interpretation of pronouns is different from adults' bidirectional interpretation, a DPBE is predicted for pronouns in German transitive sentences.

Tableau 24: Selection of the optimal form-meaning pair for the German sentence "[SD [TD Bert hasst - ]]" (Bert hates -), according to the revised model.

\begin{tabular}{|c|c|c|c|c|c|}
\hline & Bidirectional & Pr. $A_{S D}$ & *Pronoun & Pr. $A_{T D}$ & *Reflexive \\
\hline 急 & $<$ reflexive, co-referential meaning $>$ & & & & $*$ \\
\hline $\mathrm{X}$ & $<$ reflexive, disjoint meaning $>$ & * & & $*$ & $*$ \\
\hline $\mathrm{X}$ & $<$ pronoun, co-referential meaning $>$ & & * & & \\
\hline 急 & $<$ pronoun, disjoint meaning $>$ & & $*$ & & \\
\hline
\end{tabular}

As far as we know, no study has yet investigated the DPBE in German. However, our model predicts such comprehension delays to be present both for object pronouns in simple transitive sentences and for object pronouns in locational PPs.

\subsection{A DPBE in Italian transitive sentences?}

Fischer (2004) argues that the pattern of binding in Italian can be obtained using the same constraints as for English, German and Dutch, but under a different ranking. For German and Dutch, we suggested (section 4.3) that in many cases a SE anaphor and a SELF anaphor are both possible, and that the choice between the two may be influenced by factors such as focus. Italian, however, differs from German and Dutch in one crucial aspect: In Italian, SE anaphors can be realized as clitics preceding the finite verb, whereas SELF anaphors must be realized in a position following the finite verb:

(22) $\mathrm{Bert}_{\mathrm{i}} \mathrm{Si}_{\mathrm{i}}$ odia/odia se $\mathrm{stesso}_{\mathrm{i}} / * \mathrm{lo}_{\mathrm{i}}$ odia

Bert $_{i} S E_{i}$ hates/hates $S E L F_{i} / h_{i m}$ hates

"Bert hates himself" 
This difference is ignored by Fischer but may be highly relevant for the present discussion. In fact, it suggests that an additional constraint is at work in Italian, which syntactically distinguishes between SE and SELF anaphors.

Another well-known observation is that Italian and other Romance languages do not exhibit a DPBE (e.g., McKee, 1992). In her study, McKee focuses on SE anaphors and pronouns in pre-verbal clitic position in Italian and finds that, compared to English, Italian speaking children do not exhibit a DPBE with respect to the pre-verbally occurring pronoun. A pre-verbally occurring pronoun can only refer to some other individual than the local subject, also for children. From the perspective of our optimality-theoretic model, this is surprising because our model assumes that pronouns are underspecified with respect to their interpretation. Only in bidirectional competition with reflexives is one of the potential meanings blocked. So why are pre-verbally occurring pronouns in Italian not ambiguous for children?

Here, we wish to speculate on a possible explanation for the absence of a DPBE in Italian. Spenader, Smits and Hendriks (2006), in their study with Dutch speaking children, found that altering the information structure of the context had a significant effect on the presence of a DPBE in Dutch. In the standard task used by, e.g., Chien and Wexler (1990), the two referents introduced in the context are equally salient ("This is Mama Bear. This is Goldilocks"). This gives rise to a pronoun interpretation problem, also in Dutch. However, if the context is modified in such a way that there is only one salient referent, which is not the subject of the sentence (e.g., "This is Mama Bear"), children interpret the subsequent pronoun correctly as disjoint to the subject. So discourse cues in the form of a clear discourse topic dissolve the DPBE in Dutch.

Now suppose that the pre-verbal position in Italian is a topic position, i.e., a position in which only elements can appear which refer to the topic of the discourse. In this case, syntax would provide the necessary cues with respect to topic-hood to render the pronoun unambiguous. According to Spenader et al., integrating cues with respect to topic-hood into the model can be done by adding a constraint on comprehension which requires pronouns to refer to the discourse topic. Because of this relatively strong constraint ProTop ("Pronouns refer to topics"), the pronoun is interpreted as the discourse topic under unidirectional optimization (Tableau 25). 
Tableau 25: Selection of the optimal meaning for the Italian sentence "[SD [TD Bert lo odia]]" (Bert hates him) in a single-topic context where Bert $\neq$ topic), according to the revised model.

\begin{tabular}{|l|c|c|c|c|}
\hline Comprehension & Pr. $\mathrm{A}_{\mathrm{SD}}$ & ProTop & *Pronoun Pr. $\mathrm{A}_{\mathrm{TD}}$ & *Reflexive \\
\hline co-referential meaning & & $* !$ & $*$ & \\
\hline$\leftarrow$ disjoint meaning & & & $*$ & \\
\hline
\end{tabular}

If the local subject is not the discourse topic, the co-referential meaning violates the constraint ProTop. As a result, the pronoun is correctly interpreted as disjoint to the local subject. Because the output of unidirectional comprehension of preverbal pronouns in a single-topic context and the adult pattern of comprehension are the same, no DPBE arises for a pronoun in pre-verbal position.

If this explanation is correct, we predict that pronouns in post-verbal position in Italian do give rise to a DPBE because the post-verbal position is not a topic position. In the absence of a single-topic context, no cues are provided as to the topic-hood of potential referents. Consequently, pronouns in post-verbal position remain ambiguous until one of their meanings is blocked through bidirectional optimization. Baauw and Delfitto (1999) mention an unpublished pilot study by Berger (1997), who finds that Italian children appear to incorrectly allow co-reference with the subject much more often in constructions containing pronouns in post-verbal position than in constructions containing pronouns in pre-verbal position. ${ }^{7}$ This is in line with the predictions of our model, although further study is needed to corroborate this initial finding. Another prediction that requires further investigation is that adding a single-topic context will have an effect on the correct interpretation of pronouns occurring post-verbally but not on pronouns occurring pre-verbally (since syntax already provides the relevant cues here).

\section{Conclusions}

In this paper, we combined Fischer's (2004) optimality-theoretic analysis of binding, which explains the observed cross-linguistic variation in binding, and Hendriks and Spenader's (2005/6) optimality-theoretic account of the acquisition of pronouns and reflexives. The resulting optimality-theoretic model al-

7 The relevant contrast is between sentence (i) and sentence (ii) (attributed by Baauw and Delfitto (1999) to Berger (1997)):

(i) Il ragazzo sta indicando lui

(ii) Il ragazzo lo sta indicando

"The boy is pointing at him"

The post-verbally occurring form is the full pronoun lui, whereas the pre-verbally occurring form is the clitic pronoun $l o$. 
lowed us to generate predictions with respect to the presence or absence of a DPBE cross-linguistically. Acquisition delays are predicted to arise as a result of a different output for unidirectional versus bidirectional optimization. Our optimality-theoretic model predicts a DPBE to be present with respect to the comprehension of pronouns in transitive sentences in English, Dutch, and German, and in locational PPs in German. In contrast, the model predicts an absence of DPBE with respect to the comprehension of pronouns in locational PPs in English and Dutch, but not in German. Also no DPBE is predicted with respect to the comprehension of pronouns in embedded clauses in English, German, Dutch and Italian. Finally, under the additional assumption that the preverbal position in Italian is a topic position, pre-verbally occurring pronouns in Italian are predicted not to give rise to a DPBE in comprehension, whereas post-verbally occurring pronouns are expected to exhibit a DPBE.

\section{$7 \quad$ References}

Aissen, J. (1999). Markedness and subject choice in Optimality Theory. Natural Language \& Linguistic Theory, 17: 673-711.

Baauw, S. \& D. Delfitto (1999). Coreference and language acquisition. In: D. Delfitto, J. Schroten \& H. de Swart (eds.): Recherches de Linguistique Française et Romanne d'Utrecht (Utrecht Studies in Romance Linguistics) XVIII, Utrecht University.

Berger, C. (1997). Research paper 'Acquisition of Syntax', Utrecht University.

Blutner, R. (2000). Some Aspects of Optimality in Natural Language Interpretation. Journal of Semantics, 17: 189-216.

Burzio, L. (1998). Anaphora and soft constraints. In: P. Barbosa et al. (eds.): Is the best good enough? Optimality and competition in syntax. Cambridge, MA: MIT Press.

Chien, Y.-C. \& K. Wexler (1990). Children's knowledge of locality conditions on binding as evidence for the modularity of syntax and pragmatics. Language Acquisition, 13: 225295.

Chomsky, N. (1981). Lectures on Government and Binding. Dordrecht: Foris.

De Villiers, J., J. Cahillane, \& E. Altreuter (2006). What can Production Reveal about Principle B? In: K.U. Deen, J. Nomura, B. Schulz \& B.D. Schwartz (eds.): The Proceedings of the Inaugural Conference on Generative Approaches to Language Acquisition North America. Honolulu, HI: University of Connecticut Occasional Papers in Linguistics 4: 89-100.

Eckardt, R. (2001). Reanalysing selbst. Natural Language Semantics, 9: 371-412.

Fischer, S. (2004). Optimal binding. Natural Language \& Linguistic Theory, 22: 481-526.

Geurts, B. (2004). Weak and strong reflexives in Dutch. In: E. Keenan \& P. Schlenker (eds.): Proceedings of the ESSLLI'04 workshop on Semantic Approaches to Binding Theory. Nancy, France.

Hendriks, P. \& J. Spenader (2005/2006). When production precedes comprehension: An optimization approach to the acquisition of pronouns. Language Acquisition, 13: 319348.

Koster, C. (1993). Errors in Anaphora Acquisition. Doctoral dissertation, Utrecht University. 
McCarthy, J.J. (2002). A thematic guide to Optimality Theory (Research Surveys in Linguistics). Cambridge: Cambridge University Press.

McKee, C. (1992). A comparison of pronouns and anaphors in Italian and English Acquisition. Language Acquisition, 2: 21-54.

Philip, W. \& P. Coopmans (1996). The Double Dutch Delay of Principle B Effect. In: A. Stringfellow, D. Cahana-Amitay, E. Hughes \& A. Zukowski (eds.): Proceedings of the 20th Boston University Conference on Language Development. Somerville, MA: Cascadilla Press, 576-587.

Prince, A. \& P. Smolensky (2004). Optimality Theory: Constraint interaction in generative Grammar. Blackwell. Also appeared as Technical Report CU-CS-696-4993, Department of Computer Science, University of Colorado at Boulder, and Technical Report TR-2, Rutgers Center for Cognitive Science, Rutgers University, New Brunswick, NJ, April 1993.

Reinhart, T. (in press). Processing or pragmatics? Explaining the coreference delay. In: T. Gibson \& N. Pearlmutter (eds.): The processing and acquisition of reference. MIT Press.

Reinhart, T. \& E. Reuland (1993). Reflexivity. Linguistic Inquiry, 24: 657-720.

Smits, E.-J., Hendriks, P. \& J. Spenader (2007). Using very large parsed corpora and judgment data to classify verb reflexivity. In: A. Branco (ed.): Anaphora: Analysis, Algorithms and Applications. 6th Discourse Anaphora and Anaphor Resolution Colloquium, DAARC 2007. LNAI (Lecture Notes in Artifical Intelligence) \#4410, Berlin Heidelberg: Springer-Verlag, 77-93.

Spenader, J., E.-J. Smits \& P. Hendriks (2006). Coherent discourse solves the Pronoun Interpretation Problem. Ms, U. of Groningen (http://www.let.rug.nl/ hendriks/pip06.pdf).

Thornton, R. \& K. Wexler (1999). Principle B, VP Ellipsis and Interpretation in Child Grammar. Cambridge, MA: MIT Press. 